Research Article

\title{
Uniform Energy Decay Rates for the Fuzzy Viscoelastic Model with a Nonlinear Source
}

\author{
Fengyun Zhang \\ Department of Mathematics, Jining University, Qufu 273155, China \\ Correspondence should be addressed to Fengyun Zhang; yipianyun123@jnxy.edu.cn
}

Received 28 July 2020; Revised 1 September 2020; Accepted 29 September 2020; Published 27 October 2020

Academic Editor: Heng Liu

Copyright (C) 2020 Fengyun Zhang. This is an open access article distributed under the Creative Commons Attribution License, which permits unrestricted use, distribution, and reproduction in any medium, provided the original work is properly cited.

This paper considers the fuzzy viscoelastic model with a nonlinear source $u_{t t}+L u+\int_{0}^{t} g(t-\zeta) \Delta u(\zeta) \mathrm{d} \zeta-|u|^{c} u-\eta \Delta u_{t}=0$ in a bounded field $\Omega$. Under weak assumptions of the function $g(t)$, with the aid of Mathematica software, the computational technique is used to construct the auxiliary functionals and precise priori estimates. As time goes to infinity, we prove that the solution is global and energy decays to zero in two different ways: the exponential form and the polynomial form.

\section{Introduction}

In this paper, we take the following fuzzy viscoelastic model into account:

$$
\begin{cases}u_{t t}+L u+\int_{0}^{t} g(t-\zeta) \Delta u(\zeta) \mathrm{d} \zeta-|u|^{\gamma} u-\eta \Delta u_{t}=0, & x \in \Omega, t \in(0, \infty), \\ u(x, t)=0, & (x, t) \in \Gamma \times(0, \infty), \\ \left.u(x, t)\right|_{t=0}=u_{0}(x),\left.u_{t}(x, t)\right|_{t=0}=u_{1}(x), & x \in \Omega,\end{cases}
$$

where the fuzzy number $\eta \in(1,1000), \gamma>0$ :

$$
L u(t)=-\Delta u(t)+c^{2} u(t)
$$

In $R^{N}(N \geq 1), \Omega$ is a domain which is well bounded. Besides, the boundary of $\Omega$ is smooth perfectly and expressed as $\Gamma$ : $=\partial \Omega$. Meanwhile, the memory kernel $g(t)$ is positive and some assumptions will be given in detail.

This type of problems has been observed in many areas of scientific and engineering fields. For example, time analyticity for the viscoelastic equation is studied as follows [1]:

$$
u_{t t}-\Delta u+\int_{0}^{t} g(t-\zeta) \Delta u(\zeta) \mathrm{d} \zeta=|u|^{\gamma} u
$$

S. Berrimi and S. A. Messaoudi applied weak conditions on the memory kernel $g$. Meanwhile, considering the condition that the energy is positive and relatively small, they obtained the existence of global solutions.

Taking

$$
u_{t t}-\Delta u+\int_{0}^{t} g(t-\zeta) \Delta u(\zeta) \mathrm{d} \zeta+a(x) u_{t}+|u|^{\gamma} u=0
$$

into account, they also obtained a decay rate exponentially in [2]. M.M. Cavalcanti and H.P. Oquendo improved this latter result in [3]. In their work, two situations, the internal dissipation and the viscoelastic dissipation, are considered to act on respective part separately. The authors in [3] expanded the internal dissipation to nonlinear cases as much as possible. Simultaneously, the system is well stabilized through the dissipation which is induced by the integral term.

As we know, the viscoelastic terms attract many mathematicians; for instance, the authors in [4] studied the energy decay rate for the global solution of a quasilinear viscoelastic model, and the authors in [5-9] considered time analysis of solutions for some viscoelastic models. Moreover, F. Y. Zhang et al. considered a nonlinear viscoelastic equation in [10]:

$$
u_{t t}+L u+\int_{0}^{t} g(t-\zeta) \Delta u(\zeta) \mathrm{d} \zeta=0
$$


The solution is perfectly stabilized through the dissipation, which is induced by the viscoelastic term. The modified energy functional in [10] has been used to prove the energy decay through two different ways: the exponential form and the polynomial form. Additionally, the construction of auxiliary functions is organized by the computational technique of undetermined coefficients. Besides, the authors in $[11,12]$ discussed the adaptive fuzzy control of nonlinear systems, and the discussion of fuzzy coefficients involved in these papers is quite interesting.

Inspired by these works, we consider (1) in this paper, the two optimal decay rates, exponential decay and polynomial decay, are easily and directly established through the application of Mathematica software. The specific arrangement of this work is as follows: in Section 2, we present some notations and necessary materials; in Section 3 , in view of the fuzzy number $\eta$, we give the whole decay result, and our choice of the "Lyapunov" functional shows the extensive applicability and practical significance of the computational technique.

\section{Preliminaries}

In this section, $L^{p}(\Omega)$ and $H_{0}^{1}(\Omega)$ are understood and applied in their usual senses. We impose the following hypotheses and preliminaries on the memory kernel $g(t)$. In addition, the definition of energy function plays a significant role to our main result:

$\mathrm{H}_{1}$ : as a bounded $C^{1}$-function, $g(t): R_{+} \longrightarrow R_{+}$ satisfies

$$
1-\int_{0}^{\infty} g(\zeta) \mathrm{d} \zeta=\ell
$$

where both $g(0)$ and $\ell$ are positive.

$\mathrm{H}_{2}$ : the existence of a positive constant $\xi$ makes the following formula hold:

$$
g^{\prime}(t) \leq-\xi \cdot g^{p}(t), \quad t \geq 0, p \in\left[1, \frac{3}{2}\right) .
$$

Remark 1. From the assumption above, if $p=1$, we have

$$
g(t) \leq c e^{-\xi t} .
$$

If $1<p<(3 / 2)$, we have

$$
g(t) \leq \frac{1}{\left[g^{1-p}(0)+(p-1) \xi t\right]^{(1 / p-1)}} \triangleq \frac{1}{\left[d_{1} t+d_{2}\right]^{(1 / p-1)}},
$$

where $d_{1}=(p-1) \xi>0$ and $d_{2}=g^{1-p}(0)$.

Indeed, the condition $p<(3 / 2)$ plays an important role to ensure that

$$
\int_{0}^{\infty} g^{2-p}(\zeta) \mathrm{d} \zeta<\infty
$$

Theorem 1. Assuming that $\left(u_{0}, u_{1}\right) \in\left[H^{2}(\Omega) \cap H_{0}^{1}(\Omega)\right] \times$ $H_{0}^{1}(\Omega)$, then $u(t)$ can be found as a unique solution to model (1) with

$$
\begin{aligned}
u & \in L_{\mathrm{loc}}^{\infty}\left(0, \infty ; H_{0}^{1}(\Omega) \cap H^{2}(\Omega)\right), \\
u^{\prime} & \in L_{\mathrm{loc}}^{\infty}\left(0, \infty ; H_{0}^{1}(\Omega)\right), u^{\prime \prime} \in L_{\mathrm{loc}}^{\infty}\left(0, \infty ; L^{2}(\Omega)\right) .
\end{aligned}
$$

Simultaneously, we get

$$
u \in C\left([0, \infty) ; H_{0}^{1}(\Omega)\right), u^{\prime} \in C^{1}\left([0, \infty) ; L^{2}(\Omega)\right) .
$$

Taking the initial data, less regularity, and the priori estimate into consideration, the theorem above guarantees the existence of solutions for model $(1)$ as in $[7,13]$. In addition, the Galerkin approximation method can be applied to accomplish the proof of the theorem above.

Our primary assignment is to find out the energy function $\varepsilon(t)$. Combining the multiplier method, the integral subsection integration, and $\left(\mathrm{H}_{1}, \mathrm{H}_{2}\right)$, the calculation is provided:

$$
\begin{aligned}
0= & \int_{\Omega}\left(u_{t t}-\Delta u+c^{2} u+\int_{0}^{t} g(t-\zeta) \Delta u(\zeta) \mathrm{d} \zeta-|u|^{\gamma} u-\eta \Delta u_{t}\right) u_{t} \mathrm{~d} x \\
= & \frac{1}{2} \frac{\mathrm{d}}{\mathrm{d} t}\left(\int_{\Omega}\left|u_{t}\right|^{2} \mathrm{~d} x+\int_{\Omega}|\nabla u|^{2} \mathrm{~d} x+c^{2} \int_{\Omega}|u|^{2} \mathrm{~d} x\right)+\int_{0}^{t} g(t-\zeta) \int_{\Omega} \Delta u(\zeta) u_{t} \mathrm{~d} x \mathrm{~d} \zeta \\
& -\int_{\Omega}|u|^{\gamma} u u_{t} \mathrm{~d} x+\eta \int_{\Omega}\left|\nabla u_{t}\right|^{2} \mathrm{~d} x=\frac{1}{2} \frac{\mathrm{d}}{\mathrm{d} t}\left(\int_{\Omega}\left|u_{t}\right|^{2} \mathrm{~d} x+\int_{\Omega}|\nabla u|^{2} \mathrm{~d} x+c^{2} \int_{\Omega}|u|^{2} \mathrm{~d} x-\frac{1}{1+(\gamma / 2)} \int_{\Omega}|u|^{\gamma+2} \mathrm{~d} x\right) \\
& -\int_{0}^{t} g(t-\zeta) \int_{\Omega} \nabla u(\zeta) \nabla u_{t} \mathrm{~d} x \mathrm{~d} \zeta+\eta \int_{\Omega}\left|\nabla u_{t}\right|^{2} \mathrm{~d} x,
\end{aligned}
$$




$$
\begin{aligned}
& -\int_{0}^{t} g(t-\zeta) \int_{\Omega} \nabla u(\zeta) \nabla u_{t} \mathrm{~d} x \mathrm{~d} \zeta \\
& =-\int_{\Omega} \nabla u_{t} \int_{0}^{t} g(t-\zeta) \nabla u(\zeta) \mathrm{d} \zeta \mathrm{d} x \\
& =-\int_{\Omega} \nabla u_{t} \int_{0}^{t} g(t-\zeta)(\nabla u(\zeta)-\nabla u(t)) \mathrm{d} \zeta \mathrm{d} x-\int_{\Omega} \nabla u_{t} \nabla u(t) \int_{0}^{t} g(t-\zeta) \mathrm{d} \zeta \mathrm{d} x \\
& =\frac{1}{2} \frac{\mathrm{d}}{\mathrm{d} t}\left(\int_{\Omega} \int_{0}^{t} g(t-\zeta)|\nabla u(\zeta)-\nabla u(t)|^{2} \mathrm{~d} \zeta \mathrm{d} x\right) \\
& \quad-\frac{1}{2} \int_{\Omega} \int_{0}^{t} g^{\prime}(t-\zeta)|\nabla u(\zeta)-\nabla u(t)|^{2} \mathrm{~d} \zeta \mathrm{d} x-\int_{\Omega} \nabla u_{t} \nabla u(t) \int_{0}^{t} g(t-\zeta) \mathrm{d} \zeta \mathrm{d} x \\
& =\frac{1}{2} \frac{\mathrm{d}}{\mathrm{d} t}\left(g \circ \nabla u-\int_{0}^{t} g(\zeta) \mathrm{d} \zeta \int_{\Omega}|\nabla u(t)|^{2} \mathrm{~d} x\right)-\frac{1}{2}\left(g^{\prime} \circ \nabla u\right)+\frac{g(t)}{2} \int_{\Omega}|\nabla u(t)|^{2} \mathrm{~d} x .
\end{aligned}
$$

Now, (13) yields

$$
\begin{aligned}
& \frac{1}{2} \frac{\mathrm{d}}{\mathrm{d} t}\left(\int_{\Omega}\left|u_{t}\right|^{2} \mathrm{~d} x+\left(1-\int_{0}^{t} g(\zeta) \mathrm{d} \zeta\right) \int_{\Omega}|\nabla u(t)|^{2} \mathrm{~d} x\right. \\
& \left.\quad+c^{2} \int_{\Omega}|u|^{2} \mathrm{~d} x+g \circ \nabla u-\frac{\int_{\Omega}|u(t)|^{\gamma+2} \mathrm{~d} x}{1+(\gamma / 2)}\right) \\
& \quad-\frac{1}{2}\left(g^{\prime} \circ \nabla u\right)+\frac{g(t)}{2} \int_{\Omega}|\nabla u|^{2} \mathrm{~d} x+\eta \int_{\Omega}\left|\nabla u_{t}\right|^{2} \mathrm{~d} x=0 .
\end{aligned}
$$

Now, it is obvious that the energy of model (1) can be given directly:

$$
\begin{aligned}
\varepsilon(t)= & \frac{1}{2} \int_{\Omega}\left|u_{t}\right|^{2} \mathrm{~d} x+\frac{1}{2}\left(1-\int_{0}^{t} g(\zeta) \mathrm{d} \zeta\right) \int_{\Omega}|\nabla u(t)|^{2} \mathrm{~d} x \\
& +\frac{c^{2}}{2} \int_{\Omega}|u|^{2} \mathrm{~d} x+\frac{1}{2} g \circ \nabla u-\frac{\int_{\Omega}|u(t)|^{\gamma+2} \mathrm{~d} x}{\gamma+2},
\end{aligned}
$$

where

$$
g \circ f=\int_{\Omega} \int_{0}^{t} g(t-\zeta)|f(t)-f(\zeta)|^{2} \mathrm{~d} \zeta \mathrm{d} x .
$$

Thereby, we can find

$$
\begin{aligned}
\mathcal{E}^{\prime}(t) & =\frac{\mathrm{d}}{\mathrm{d} t} \varepsilon(t) \\
& =\frac{1}{2}\left(\left(g^{\prime} \circ \nabla u\right)-\int_{\Omega}|\nabla u|^{2} \mathrm{~d} x\right)-\eta \int_{\Omega}\left|\nabla u_{t}\right|^{2} \mathrm{~d} x \\
& \leq \frac{1}{2}\left(\left(g^{\prime} \circ \nabla u\right)-\int_{\Omega}|\nabla u|^{2} \mathrm{~d} x\right) \\
& \leq \frac{1}{2}\left(-\xi\left(g^{p} \circ \nabla u\right)-g(t) \int_{\Omega}|\nabla u|^{2} \mathrm{~d} x\right) \\
& \leq 0,
\end{aligned}
$$

which means $\varepsilon(t)$ is decreasing.

\section{Main Results}

In this chapter, the main result of this work is put forward directly and clearly. We introduce the following auxiliary functionals firstly:

$$
\begin{aligned}
& \varphi(t)=\int_{\Omega} u_{t} u \mathrm{~d} x+\int_{\Omega}|\nabla u|^{2} \mathrm{~d} x, \\
& \chi(t)=\int_{\Omega}\left(u+\alpha \Delta u-\beta u_{t}\right) \int_{0}^{t} g(t-\zeta)(u(t)-u(\zeta)) \mathrm{d} \zeta \mathrm{d} x,
\end{aligned}
$$

where the coefficients $\alpha$ and $\beta$ will be determined later.

Lemma 1. The existence of a positive constant $C$ makes the following conclusion:

$$
|\varphi(t)| \leq C \varepsilon(t), \quad \forall t \geq 0 .
$$

Proof. Recalling the inequalities of Cauchy and Poincaré, the following estimates can be arrived:

$$
\begin{aligned}
|\varphi(t)| & \leq \frac{\int_{\Omega}\left|u_{t}\right|^{2} \mathrm{~d} x+\int_{\Omega}|u|^{2} \mathrm{~d} x}{2}+\int_{\Omega}|\nabla u|^{2} \mathrm{~d} x \\
& \leq \frac{C_{p}+1}{2} \int_{\Omega}|\nabla u|^{2} \mathrm{~d} x+\frac{1}{2} \int_{\Omega}\left|u_{t}\right|^{2} \mathrm{~d} x,
\end{aligned}
$$

where the symbol $C_{p}>0$ satisfies $\|u\|^{2} \leq C_{p}\|\nabla u\|^{2}$ for all $u \in H_{0}^{1}(\Omega)$.

Here, it is necessary to indicate that the positive constants $C$ and $C_{p}$ later in this article represent different meanings in different places.

From the representation of $\varepsilon(t)$, it is naturally for us to get

$$
|\varphi(t)| \leq C \varepsilon(t), \quad \forall t \geq 0 .
$$

Thus, (20) follows.

Lemma 2. For any $t \geq 0$, the following estimate holds: 


$$
\begin{aligned}
\varphi^{\prime}(t) \leq & \int_{\Omega}\left|u_{t}\right|^{2} \mathrm{~d} x+\left(\frac{1}{4 \varepsilon_{1}}+\varepsilon_{1}\left(1+\frac{1}{2 \varepsilon_{2}}\right)(1-\ell)^{2}\right. \\
& \left.+\frac{C_{p}^{2 \gamma+2}}{2}\left(\frac{(2 \gamma+4) E(0)}{\gamma \ell}\right)^{\gamma}-\eta\right) \int_{\Omega}|\nabla u|^{2} \mathrm{~d} x \\
& +\left(\frac{1}{2}-c^{2}\right) \int_{\Omega}|u|^{2} \mathrm{~d} x+\varepsilon_{1}(1 \\
& \left.+2 \varepsilon_{2}\right)\left(\int_{0}^{t} g^{2-p}(\zeta) \mathrm{d} \zeta\right) g^{p} \circ \nabla u+\frac{2-\eta}{2} \int_{\Omega}\left|\nabla u_{t}\right|^{2} \mathrm{~d} x
\end{aligned}
$$

where $\varepsilon_{1}$ and $\varepsilon_{2}$ are selected appropriately.
Proof. Deriving function $\varphi(t)$ with respect to time, the following calculation will be given clearly:

$$
\begin{aligned}
\frac{\mathrm{d}}{\mathrm{d} t} \varphi(t)= & \int_{\Omega}\left|u_{t}\right|^{2} \mathrm{~d} x+\int_{\Omega} u_{t t} u \mathrm{~d} x+2 \int_{\Omega} \nabla u \nabla u_{t} \mathrm{~d} x \\
= & \int_{\Omega}\left|u_{t}\right|^{2} \mathrm{~d} x-\int_{\Omega}|\nabla u|^{2} \mathrm{~d} x-c^{2} \int_{\Omega}|u|^{2} \mathrm{~d} x \\
& +\int_{\Omega} \int_{0}^{t} g(t-\zeta) \nabla u(t) \nabla u(\zeta) \mathrm{d} \zeta \mathrm{d} x \\
& +\int_{\Omega}|u|^{\gamma} u^{2} \mathrm{~d} x+(2-\eta) \int_{\Omega} \nabla u \nabla u_{t} \mathrm{~d} x .
\end{aligned}
$$

Considering the fourth item of (23), we adopt the following estimate from [10] without proof:

$$
\begin{aligned}
& \int_{\Omega} \int_{0}^{t} g(t-\zeta) \nabla u(t) \nabla u(\zeta) \mathrm{d} \zeta \mathrm{d} x \\
& \leq\left(\frac{1}{4 \varepsilon_{1}}+\varepsilon_{1}\left(1+\frac{1}{2 \varepsilon_{2}}\right)(1-\ell)^{2}\right) \int_{\Omega}|\nabla u|^{2} \mathrm{~d} x+\varepsilon_{1}\left(1+2 \varepsilon_{2}\right)\left(\int_{0}^{t} g^{2-p}(\zeta) \mathrm{d} \zeta\right) g^{p} \circ \nabla u
\end{aligned}
$$

Taking the sixth item of (23) into account, we adopt the following estimate from [1] as follows:

$$
\begin{aligned}
\int_{\Omega}|u|^{\gamma} u^{2} \mathrm{~d} x & =\int_{\Omega}|u|^{\gamma+1} u \mathrm{~d} x \\
& \leq \frac{1}{2} \int_{\Omega}|u|^{2(\gamma+1)} \mathrm{d} x+\frac{1}{2} \int_{\Omega}|u|^{2} \mathrm{~d} x \\
& \leq \frac{C_{p}^{2 \gamma+2}}{2}\left(\frac{(2 \gamma+4) E(0)}{\gamma \ell}\right)^{\gamma} \int_{\Omega}|\nabla u|^{2} \mathrm{~d} x+\frac{1}{2} \int_{\Omega}|u|^{2} \mathrm{~d} x .
\end{aligned}
$$

For the last item of (23), we have

$$
(2-\eta) \int_{\Omega} \nabla u \nabla u_{t} \mathrm{~d} x \leq\left(1-\frac{\eta}{2}\right)\left(\int_{\Omega}|\nabla u|^{2} \mathrm{~d} x+\int_{\Omega}\left|\nabla u_{t}\right|^{2} \mathrm{~d} x\right) .
$$

Thus, (21) follows.

Lemma 3. For $t \geq 0$, the following estimate holds:

$$
\begin{aligned}
\chi^{\prime}(t) \leq & \left(\beta c^{2} \varepsilon_{6}+\varepsilon_{9}+(1-\ell) \varepsilon_{12}\right) \int_{\Omega}|u|^{2} \mathrm{~d} x+\left(\varepsilon_{3}+\beta \varepsilon_{11}+\left(\frac{1}{4 \varepsilon_{12}}-\beta\right)(1-\ell)\right) \int_{\Omega}\left|u_{t}\right|^{2} \mathrm{~d} x \\
& +\left(\frac{C_{p}}{4 \varepsilon_{3}}+\frac{\beta \eta-\alpha}{4 \varepsilon_{4}}+\frac{c^{2} \beta}{4 \varepsilon_{6}}+\beta\left(\varepsilon_{7}+\frac{1}{4 \varepsilon_{7}}\right)+\frac{\beta}{4 \varepsilon_{8}}\right)\left(\int_{0}^{t} g^{2-p}(\zeta) \mathrm{d} \zeta\right) g^{p} \circ \nabla u \\
& +g(0)\left(\frac{C_{p}}{4 \varepsilon_{9}}+\frac{\alpha}{4 \varepsilon_{10}}+\frac{\beta C_{p}}{4 \varepsilon_{11}}\right)\left(-g^{\prime} \circ \nabla u\right)+\left(\beta \varepsilon_{5}+\beta \varepsilon_{7}(1-\ell)^{2}+\beta \varepsilon_{8} C_{p}^{2 \gamma+2}\left(\frac{(2 \gamma+4) E(0)}{\gamma \ell}\right)^{\gamma}+\alpha \varepsilon_{10}+\alpha \varepsilon_{13}(1-\ell)\right) \int_{\Omega}|\nabla u|^{2} \mathrm{~d} x \\
& +\left(\varepsilon_{4}(\beta \eta-\alpha)+\frac{\alpha}{4 \varepsilon_{13}}\right) \int_{\Omega}\left|\nabla u_{t}\right|^{2} \mathrm{~d} x,
\end{aligned}
$$


Proof. Deriving function $\chi(t)$ with respect to time, the expression will be given distinctly:

$$
\begin{aligned}
& \frac{\mathrm{d}}{\mathrm{d} t} \chi(t) \\
& =\int_{\Omega}\left(u_{t}+\alpha \Delta u_{t}-\beta u_{t t}\right) \int_{0}^{t} g(t-\zeta)(u(t)-u(\zeta)) \mathrm{d} \zeta \mathrm{d} x \\
& +\int_{\Omega}\left(u+\alpha \Delta u-\beta u_{t}\right) \int_{0}^{t} g^{\prime}(t-\zeta)(u(t)-u(\zeta)) \mathrm{d} \zeta \mathrm{d} x+\int_{\Omega}\left(u+\alpha \Delta u-\beta u_{t}\right) \int_{0}^{t} g(t-\zeta) u_{t} \mathrm{~d} \zeta \mathrm{d} x \\
& =\int_{\Omega}\left(u_{t}+\alpha \Delta u_{t}-\beta\left(-\Delta u+c^{2} u+\int_{0}^{t} g(t-\zeta) \Delta u(\zeta) \mathrm{d} \zeta-|u|^{\gamma} u-\eta \Delta u_{t}\right)\right)\left(\int_{0}^{t} g(t-\zeta)(u(t)-u(\zeta)) \mathrm{d} \zeta\right) \mathrm{d} x \\
& +\int_{\Omega}\left(u+\alpha \Delta u-\beta u_{t}\right) \int_{0}^{t} g^{\prime}(t-\zeta)(u(t)-u(\zeta)) \mathrm{d} \zeta \mathrm{d} x+\left(\int_{0}^{t} g(\zeta) \mathrm{d} \zeta\right) \int_{\Omega}\left(u+\alpha \Delta u-\beta u_{t}\right) u_{t} \mathrm{~d} x \\
& =\int_{\Omega}\left(u_{t}+(\alpha-\beta \eta) \Delta u_{t}-\beta \Delta u+\beta c^{2} u+\beta \int_{0}^{t} g(t-\zeta) \Delta u(\zeta) \mathrm{d} \zeta-\beta|u|^{\gamma} u\right)\left(\int_{0}^{t} g(t-\zeta)(u(t)-u(\zeta)) \mathrm{d} \zeta \mathrm{d} x\right) \\
& +\int_{\Omega}\left(u+\alpha \Delta u-\beta u_{t}\right) \int_{0}^{t} g^{\prime}(t-\zeta)(u(t)-u(\zeta)) \mathrm{d} \zeta \mathrm{d} x+\left(\int_{0}^{t} g(\zeta) \mathrm{d} \zeta\right) \int_{\Omega}\left(u+\alpha \Delta u-\beta u_{t}\right) u_{t} \mathrm{~d} x \\
& =\int_{\Omega} u_{t}\left(\int_{0}^{t} g(t-\zeta)(u(t)-u(\zeta)) \mathrm{d} \zeta\right) \mathrm{d} x \\
& +\int_{\Omega} \nabla u_{t}(t)(\beta \eta-\alpha) \int_{0}^{t} g(t-\zeta)(\nabla u(t)-\nabla u(\zeta)) \mathrm{d} \zeta \mathrm{d} x \\
& +\beta \int_{\Omega} \nabla u \int_{0}^{t} g(t-\zeta)(\nabla u(t)-\nabla u(\zeta)) \mathrm{d} \zeta \mathrm{d} x \\
& +\beta c^{2} \int_{\Omega} u \int_{0}^{t} g(t-\zeta)(u(t)-u(\zeta)) \mathrm{d} \zeta \mathrm{d} x \\
& +\beta\left(\int_{0}^{t} g(t-\zeta) \Delta u(\zeta) \mathrm{d} \zeta\right)\left(\int_{0}^{t} g(t-\zeta)(u(t)-u(\zeta)) \mathrm{d} \zeta\right) \mathrm{d} x \\
& -\beta \int_{\Omega}|u|^{1+\gamma} \int_{0}^{t} g(t-\zeta)(u(t)-u(\zeta)) \mathrm{d} \zeta \mathrm{d} x \\
& +\int_{\Omega}\left(u+\alpha \Delta u-\beta u_{t}\right) \int_{0}^{t} g^{\prime}(t-\zeta)(u(t)-u(\zeta)) \mathrm{d} \zeta \mathrm{d} x+\left(\int_{0}^{t} g(\zeta) \mathrm{d} \zeta\right) \int_{\Omega}\left(u u_{t}+\alpha u_{t} \Delta u-\beta u_{t}^{2}\right) \mathrm{d} x
\end{aligned}
$$


For any $\alpha, \beta \geq 0$, we will estimate every item of the right hand of (29):

$$
\begin{aligned}
& \int_{\Omega} u_{t} \int_{0}^{t} g(t-\zeta)(u(t)-u(\zeta)) \mathrm{d} \zeta \mathrm{d} x \\
& \quad \leq \varepsilon_{3} \int_{\Omega}\left|u_{t}\right|^{2} \mathrm{~d} x+\frac{1}{4 \varepsilon_{3}} \int_{\Omega}\left|\int_{0}^{t} g(t-\zeta)(u(t)-u(\zeta)) \mathrm{d} \zeta\right|^{2} \mathrm{~d} x \\
& \quad=\varepsilon_{3} \int_{\Omega}\left|u_{t}\right|^{2} \mathrm{~d} x+\frac{1}{4 \varepsilon_{3}} \int_{\Omega}\left|\int_{0}^{t} g^{1-(p / 2)}(t-\zeta) g^{(p / 2)}(t-\zeta)(u(t)-u(\zeta)) \mathrm{d} \zeta\right|^{2} \mathrm{~d} x \\
& \quad \leq \varepsilon_{3} \int_{\Omega}\left|u_{t}\right|^{2} \mathrm{~d} x+\frac{C_{p}}{4 \varepsilon_{3}}\left(\int_{0}^{t} g^{2-p}(\zeta) \mathrm{d} \zeta\right)\left(g^{p} \circ \nabla u\right) \\
& (\beta \eta-\alpha) \int_{\Omega} \nabla u_{t}(t) \int_{0}^{t} g(t-\zeta)(\nabla u(t)-\nabla u(\zeta)) \mathrm{d} \zeta \mathrm{d} x \\
& \quad \leq(\beta \eta-\alpha)\left(\varepsilon_{4} \int_{\Omega}\left|\nabla u_{t}\right|^{2} \mathrm{~d} x+\frac{1}{4 \varepsilon_{4}} \int_{\Omega}\left(\int_{0}^{t} g(t-\zeta)|\nabla u(t)-\nabla u(\zeta)| \mathrm{d} \zeta\right)^{2} \mathrm{~d} x\right) \\
& \quad \leq(\beta \eta-\alpha) \varepsilon_{4} \int_{\Omega}\left|\nabla u_{t}\right|^{2} \mathrm{~d} x+\frac{\beta \eta-\alpha}{4 \varepsilon_{4}}\left(\int_{0}^{t} g^{2-p}(\zeta) \mathrm{d} \zeta\right)\left(g^{p} \circ \nabla u\right), \\
& \quad \nabla u \int_{0}^{t} g(t-\zeta)(\nabla u(t)-\nabla u(\zeta)) \mathrm{d} \zeta \mathrm{d} x \leq \beta \varepsilon_{5} \int_{\Omega}|\nabla u|^{2} \mathrm{~d} x+\frac{\beta}{4 \varepsilon_{5}}\left(\int_{0}^{t} g^{2-p}(\zeta) \mathrm{d} \zeta\right)\left(g^{p} \circ \nabla u\right)
\end{aligned}
$$

Similar to the first term, considering the third item of (29), similar estimate will be given:

$$
\begin{aligned}
& \beta c^{2} \int_{\Omega} u \int_{0}^{t} g(t-\zeta)(\nabla u(t)-\nabla u(\zeta)) \mathrm{d} \zeta \mathrm{d} x \\
& \quad \leq \beta c^{2} \varepsilon_{6} \int_{\Omega}|u|^{2} \mathrm{~d} x+\frac{\beta c^{2}}{4 \varepsilon_{6}}\left(\int_{0}^{t} g^{2-p}(\zeta) \mathrm{d} \zeta\right)\left(g^{p} \circ \nabla u .\right)
\end{aligned}
$$

Applying Young's inequality properly, the following estimates can be obtained from [10]:

$$
\begin{aligned}
& \beta\left(\int_{0}^{t} g(t-\zeta) \Delta u(\zeta) \mathrm{d} \zeta\right)\left(\int_{0}^{t} g(t-\zeta)(u(t)-u(\zeta)) \mathrm{d} \zeta\right) \mathrm{d} x \\
& \quad=\beta\left(\int_{0}^{t} g(t-\zeta) \nabla u(\zeta) \mathrm{d} \zeta\right)\left(\int_{0}^{t} g(t-\zeta)(\nabla u(\zeta)-\nabla u(t)) \mathrm{d} \zeta\right) \mathrm{d} x \\
& \quad \leq \beta(1-\ell)^{2} \varepsilon_{7} \int_{\Omega}|\nabla u|^{2} \mathrm{~d} x+\beta\left(\varepsilon_{7}+\frac{1}{4 \varepsilon_{7}}\right)\left(\int_{0}^{t} g^{2-p}(\zeta) \mathrm{d} \zeta\right)\left(g^{p} \circ \nabla u\right)
\end{aligned}
$$




$$
\begin{aligned}
& -\beta \int_{\Omega}|u|^{\gamma} u \int_{0}^{t} g(t-\zeta)(u(t)-u(\zeta)) \mathrm{d} \zeta \mathrm{d} x \\
& \leq \beta \varepsilon_{8} \int_{\Omega}|u|^{2 \gamma+2} \mathrm{~d} x+\left.\frac{\beta}{4 \varepsilon_{8}} \int_{\Omega}\left(\int_{0}^{t} g(t-\zeta) \mid u(t)-u(\zeta)\right) d \zeta\right|^{2} \mathrm{~d} x \\
& \leq \beta \varepsilon_{8} C_{p}^{2 \gamma+2}\left(\frac{(2 \gamma+4) E(0)}{\gamma \ell}\right) \int_{\Omega}^{\gamma}|\nabla u|^{2} \mathrm{~d} x+\frac{\beta}{4 \varepsilon_{8}}\left(\int_{0}^{t} g^{2-p}(\zeta) \mathrm{d} \zeta\right)\left(g^{p} \circ \nabla u\right), \\
& \int_{\Omega} u \int_{0}^{t} g^{\prime}(t-\zeta)(u(t)-u(\zeta)) \mathrm{d} \zeta \mathrm{d} x \\
& \quad \leq \varepsilon_{9} \int_{\Omega}|u|^{2} \mathrm{~d} x+\frac{g(0) C_{p}}{4 \varepsilon_{9}} \int_{\Omega}\left(\int_{0}^{t}-g^{\prime}(t-\zeta)|\nabla u(t)-\nabla u(\zeta)|^{2} \mathrm{~d} \zeta\right) \mathrm{d} x \\
& \quad=\varepsilon_{9} \int_{\Omega}|u|^{2} \mathrm{~d} x+\frac{g(0) C_{p}}{4 \varepsilon_{9}}\left(-g^{\prime} \circ \nabla u\right), \\
& \alpha \int_{\Omega} \Delta u \int_{0}^{t} g^{\prime}(t-\zeta)(u(t)-u(\zeta)) \mathrm{d} \zeta \mathrm{d} x \\
& \quad \leq \alpha \varepsilon_{10} \int_{\Omega}|\nabla u|^{2} d x+\frac{\alpha g(0)}{4 \varepsilon_{10}} \int_{\Omega}\left(\int_{0}^{t}-g^{\prime}(t-\zeta)|\nabla u(t)-\nabla u(\zeta)|^{2} \mathrm{~d} \zeta\right) \mathrm{d} x \\
& =\alpha \varepsilon_{10} \int_{\Omega}|\nabla u|^{2} d x+\frac{\alpha g(0)}{4 \varepsilon_{10}}\left(-g^{\prime} \circ \nabla u\right) .
\end{aligned}
$$

Similarly, we have

Furthermore,

$$
\begin{aligned}
& -\beta \int_{\Omega} u_{t} \int_{0}^{t} g^{\prime}(t-\zeta)(u(t)-u(\zeta)) \mathrm{d} \zeta \mathrm{d} x \\
& \quad \leq \beta \varepsilon_{11} \int_{\Omega}\left|u_{t}\right|^{2} \mathrm{~d} x+\frac{\beta g(0) C_{p}}{4 \varepsilon_{11}}\left(-g^{\prime} \circ \nabla u\right) .
\end{aligned}
$$

$$
\begin{aligned}
& \left(\int_{0}^{t} g(\zeta) \mathrm{d} \zeta\right) \int_{\Omega}\left(u u_{t}+\alpha u_{t} \Delta u-\beta u_{t}^{2}\right) \mathrm{d} x \\
& \quad \leq\left(\int_{0}^{t} g(\zeta) \mathrm{d} \zeta\right)\left(\varepsilon_{12} \int_{\Omega}|u|^{2} \mathrm{~d} x+\left.\left(\frac{1}{4 \varepsilon_{12}}-\beta\right) \int_{\Omega}\left|u_{t}\right|^{2} \mathrm{~d} x\left|+\alpha \varepsilon_{13} \int_{\Omega}\right| \nabla u\right|^{2} \mathrm{~d} x+\frac{\alpha}{4 \varepsilon_{13}} \int_{\Omega}\left|\nabla u_{t}\right|^{2} \mathrm{~d} x\right) \\
& \quad=(1-\ell)\left(\varepsilon_{12} \int_{\Omega}|u|^{2} \mathrm{~d} x+\left(\frac{1}{4 \varepsilon_{12}}-\beta\right) \int_{\Omega}\left|u_{t}\right|^{2} \mathrm{~d} x+\alpha \varepsilon_{13} \int_{\Omega}|\nabla u|^{2} \mathrm{~d} x+\frac{\alpha}{4 \varepsilon_{13}} \int_{\Omega}\left|\nabla u_{t}\right|^{2} \mathrm{~d} x\right)
\end{aligned}
$$

Taking (29)-(39) and $\mathrm{H}_{2}$ into account, for all $t \geq 0$, we get (28).

Theorem 2. Let every pair $\left(u_{0}, u_{1}\right) \in H_{0}^{1}(\Omega) \times H_{0}^{1}(\Omega), H_{1}$ and $\mathrm{H}_{2}$ hold, and $t_{0} \in(0, \infty)$ For every $t \in\left[t_{0}, \infty\right)$, there must be some positive constants $K_{1}, K_{2}$, and $k$, which would enable the solution of the model (1) to satisfy the following:

$$
\begin{aligned}
& \varepsilon(t) \leq \frac{K_{1} \varepsilon(0)}{e^{k t}}, \quad p=1, \\
& \varepsilon(t) \leq \frac{K_{2}}{(t+1)^{(1 / 2(p-1))}}, \quad p>1 .
\end{aligned}
$$

Proof. We start the proof by selecting an appropriate auxiliary functional 


$$
L(t)=M \varepsilon(t)+a \varphi(t)+b \chi(t),
$$

where the positive constants $M, a$, and $b$ will be chosen in the sequel.
Applying Lemma 1 and the hypothesis $g^{\prime}(t) \leq-\xi \cdot g^{p}(t)$, the equivalence between $\varepsilon(t)$ and $L(t)$ is achieved. To this point, firstly, a simple calculation shows that

$$
\begin{aligned}
\mid L(t) & -M \varepsilon(t) \mid \\
= & |a \varphi(t)+b \chi(t)| \\
= & \left.\left|a \int_{\Omega} u_{t} u \mathrm{~d} x+a \int_{\Omega}\right| \nabla u\right|^{2} \mathrm{~d} x+b \int_{\Omega}\left(u+\alpha \Delta u-\beta u_{t}\right) \int_{0}^{t} g(t-\zeta)(u(t)-u(\zeta)) \mathrm{d} \zeta \mathrm{d} x \mid \\
= & \left.\left|a \int_{\Omega} u_{t} u \mathrm{~d} x+a \int_{\Omega}\right| \nabla u\right|^{2} \mathrm{~d} x+b \int_{\Omega}\left(u-\beta u_{t}\right) \int_{0}^{t} g(t-\zeta)(u(t)-u(\zeta)) \mathrm{d} \zeta \mathrm{d} x-b \alpha \int_{\Omega} \nabla u \int_{0}^{t} g(t-\zeta)(u(t)-u(\zeta)) \mathrm{d} \zeta \mathrm{d} x \mid \\
\leq & \frac{|a|}{2} \int_{\Omega}\left|u_{t}\right|^{2} \mathrm{~d} x+\frac{|a| C_{p}+2 a}{2} \int_{\Omega}|\nabla u|^{2} \mathrm{~d} x+\frac{|b|}{2} \int_{\Omega}\left(u-\beta u_{t}\right)^{2} \mathrm{~d} x+\frac{|b \alpha|}{2} \int_{\Omega}|\nabla u|^{2} \mathrm{~d} x \\
& +\frac{|b|}{2} \int_{\Omega}\left(\int_{0}^{t} g(t-\zeta)(u(t)-u(\zeta)) \mathrm{d} \zeta\right)^{2} \mathrm{~d} x+\frac{|b \alpha|}{2} \int_{\Omega}\left(\int_{0}^{t} g(t-\zeta)(\nabla u(t)-\nabla u(\zeta)) \mathrm{d} \zeta\right)^{2} \mathrm{~d} x \\
\leq & \frac{|a|}{2} \int_{\Omega}\left|u_{t}\right|^{2} \mathrm{~d} x+\frac{|a| C_{p}+2 a+|b \alpha|}{2} \int_{\Omega}|\nabla u|^{2} \mathrm{~d} x+\frac{|b|}{2} \int_{\Omega}|u|^{2} \mathrm{~d} x \\
& +\frac{\beta^{2}|b|}{2} \int_{\Omega}\left|u_{t}\right|^{2} \mathrm{~d} x-\beta|b| \int_{\Omega} u_{t} u \mathrm{~d} x+\frac{|b|}{2}\left(\frac{1}{2} \int_{\Omega} \int_{0}^{t} g(t-\zeta) \mathrm{d} \zeta \mathrm{d} x+\frac{1}{2} \int_{\Omega} \int_{0}^{t} g(t-\zeta)|u(t)-u(\zeta)|^{2} \mathrm{~d} \zeta \mathrm{d} x\right) \\
& +\frac{|b \alpha|}{2}\left(\frac{1}{2} \int_{\Omega} \int_{0}^{t} g(t-\zeta) \mathrm{d} \zeta \mathrm{d} x+\frac{1}{2} \int_{\Omega} \int_{0}^{t} g(t-\zeta)|\nabla u(t)-\nabla u(\zeta)|^{2} \mathrm{~d} \zeta \mathrm{d} x\right) \\
\leq & \frac{|a|+|b| \beta^{2}}{2} \int_{\Omega}\left|u_{t}\right|^{2} \mathrm{~d} x+\frac{|a| C_{p}+2 a+|b \alpha|}{2} \int_{\Omega}|\nabla u|^{2} \mathrm{~d} x+\frac{|b|}{2} \int_{\Omega}|u|^{2} \mathrm{~d} x+\frac{|b \alpha|+|b| C_{p}}{4} g \circ \nabla u \\
& +\frac{|b|(1+|\alpha|)(1-\ell)}{4} \int_{\Omega} \mathrm{d} x \\
& C_{1} \varepsilon(t) .
\end{aligned}
$$

By (25), we get

$$
\begin{aligned}
& |L(t)-M \varepsilon(t)|+\frac{1}{\gamma+2} \int_{\Omega}|u|^{\gamma+2} \mathrm{~d} x \\
& \leq \frac{|a|+|b| \beta^{2}}{2} \int_{\Omega}\left|u_{t}\right|^{2} \mathrm{~d} x+\frac{1}{2}\left(|a| C_{p}+2 a+|b \alpha|+C_{p}^{2 \gamma+2}\left(\frac{(2 \gamma+4) E(0)}{\gamma \ell}\right)^{\gamma}\right) \int_{\Omega}|\nabla u|^{2} \mathrm{~d} x \\
& \quad+\frac{|b|+1}{2} \int_{\Omega}|u|^{2} \mathrm{~d} x+\frac{|b \alpha|+|b| C_{p}}{4} g \circ \nabla u+\frac{|b|(1+|\alpha|)(1-\ell)}{4} \int_{\Omega} \mathrm{d} x \\
& \leq C_{1}\left(\varepsilon(t)+\frac{1}{\gamma+2} \int_{\Omega}|u|^{\gamma+2} \mathrm{~d} x\right) .
\end{aligned}
$$


Thus, the equivalence between $L(t)$ and $\varepsilon(t)$ is complete.

$$
\begin{aligned}
L^{\prime}(t) & =M \varepsilon^{\prime}(t)+a \varphi^{\prime}(t)+b \chi^{\prime}(t) \\
& \leq M\left[-\frac{\xi}{2}\left(g^{p} \circ \nabla u\right)-\frac{g(t)}{2} \int_{\Omega}|\nabla u|^{2} \mathrm{~d} x\right]+a \varphi^{\prime}(t)+b \chi^{\prime}(t) \\
& \leq c_{1} \int_{\Omega}\left|u_{t}\right|^{2} \mathrm{~d} x+c_{2} \int_{\Omega}|\nabla u|^{2} \mathrm{~d} x+c_{3} \int_{\Omega}|u|^{2} \mathrm{~d} x+c_{4} g^{p} \circ \nabla u+c_{5} \int_{\Omega}\left|\nabla u_{t}\right|^{2} \mathrm{~d} x,
\end{aligned}
$$

where the coefficients $c_{1}, c_{2}, c_{3}$, and $c_{4}$ are given as follows:

$$
\begin{aligned}
c_{1}= & a+b \varepsilon_{3}+b \beta \varepsilon_{11}+b(1-\ell)\left(\frac{1}{4 \varepsilon_{12}}-\beta\right), \\
c_{2}= & a\left(\frac{1}{4 \varepsilon_{1}}+\varepsilon_{1}\left(1+\frac{1}{2 \varepsilon_{2}}\right)(1-\ell)^{2}+\frac{C_{p}^{2 \gamma+2}}{2}\left(\frac{(2 \gamma+4) E(0)}{\gamma \ell}\right)^{\gamma}-\frac{\eta}{2}\right) \\
& +b\left(\beta \varepsilon_{5}+\beta \varepsilon_{7}(1-\ell)^{2}+\beta \varepsilon_{8} C_{p}^{2 \gamma+2}\left(\frac{(2 \gamma+4) E(0)}{\gamma \ell}\right)^{\gamma}+\alpha \varepsilon_{10}+\alpha \varepsilon_{13}(1-\ell)\right)-\frac{M g(t)}{2}, \\
c_{3}= & a\left(\frac{1}{2}-c^{2}\right)+b\left(\beta c^{2} \varepsilon_{6}+\varepsilon_{9}+\varepsilon_{12}(1-\ell)\right), \\
c_{4}= & \left(a \varepsilon_{1}\left(1+2 \varepsilon_{2}\right)+b\left(\frac{C_{p}}{4 \varepsilon_{3}}+\frac{\beta \eta-\alpha}{4 \varepsilon_{4}}+\frac{c^{2} \beta}{4 \varepsilon_{6}}+\beta\left(\varepsilon_{7}+\frac{1}{4 \varepsilon_{7}}\right)+\frac{\beta}{4 \varepsilon_{8}}\right)\right)\left(\int_{0}^{t} g^{2-p}(\zeta) \mathrm{d} \zeta\right) \\
& +b \xi\left(\frac{g(0) C_{p}}{4 \varepsilon_{9}}+\frac{g(0) \alpha}{4 \varepsilon_{10}}+\frac{\beta g(0) C_{p}}{4 \varepsilon_{11}}\right)-\frac{M \xi}{2}, \\
c_{5}= & a\left(1-\frac{\eta}{2}\right)+b\left(\varepsilon_{4}(\beta \eta-\alpha)+\frac{\alpha}{\varepsilon_{13}}\right) .
\end{aligned}
$$

Next, it is time to carry out a discussion on the different values of $p$.

Case 1. $p=1$

In such a cheosing $\tilde{c_{1}}=c_{1}, \tilde{c_{2}}=c_{2}+(h / \gamma+2)\left(C_{p}^{2 \gamma+2} / 2\right)((2 \gamma+4) E(0) / \gamma \ell)^{\gamma}, \tilde{c_{3}}=$ $c_{3}+(h / 2(\gamma+2)), \tilde{c_{4}}=c_{4}, \widetilde{c_{5}}=c_{5}=0$ and taking the positive constant $h$ as

$$
h \leq \min \left\{-2 \tilde{c_{1}}, \frac{-2 \tilde{c_{2}}}{1-\int_{0}^{t} g(\zeta) \mathrm{d} \zeta}, \frac{-2 \tilde{c_{3}}}{c^{2}},-2 \tilde{c_{4}}\right\},
$$


we find

$$
\begin{aligned}
& L^{\prime}(t)-\frac{h}{\gamma+2} \int_{\Omega}|u|^{\gamma+2} \mathrm{~d} x \\
& \quad \leq L^{\prime}(t)+\frac{h}{\gamma+2} \int_{\Omega}|u|^{\gamma+2} \mathrm{~d} x \\
& \quad \leq L^{\prime}(t)+\frac{h}{\gamma+2} \frac{C_{p}^{2 \gamma+2}}{2}\left(\frac{(2 \gamma+4) E(0)}{\gamma \ell}\right)^{\gamma} \int_{\Omega}|\nabla u|^{2} \mathrm{~d} x+\frac{h}{2(\gamma+2)} \int_{\Omega}|u|^{2} \mathrm{~d} x \\
& \quad \leq \tilde{c_{1}} \int_{\Omega}\left|u_{t}\right|^{2} \mathrm{~d} x+\tilde{c_{2}} \int_{\Omega}|\nabla u|^{2} \mathrm{~d} x+\tilde{c_{3}} \int_{\Omega}|u|^{2} \mathrm{~d} x+\tilde{c_{4}} g^{p} \circ \nabla u+\tilde{c_{5}} \int_{\Omega}\left|\nabla u_{t}\right|^{2} \mathrm{~d} x \\
& \quad \leq-h\left(\frac{1}{2} \int_{\Omega}\left|u_{t}\right|^{2} \mathrm{~d} x+\frac{1}{2}\left(1-\int_{0}^{t} g(\zeta) \mathrm{d} \zeta\right) \int_{\Omega}|\nabla u|^{2} \mathrm{~d} x+\frac{c^{2}}{2} \int_{\Omega}|u|^{2} \mathrm{~d} x+\frac{1}{2} g \circ \nabla u\right) . \\
& \quad \tilde{c_{1}}=-4.85169-18.8333(1-\ell),
\end{aligned}
$$

Therefore, (45) yields

$$
L^{\prime}(t) \leq-h \mathcal{\varepsilon}(t) .
$$

Remark 2. Using the computational technique proposed in [10] and selecting some appropriate numerical values by means of the calculation software Mathematica, it is obvious that $\tilde{c_{i}}<0(1 \leq i \leq 4)$.

Considering the interval of the fuzzy number $\eta$, taking

$$
\begin{aligned}
M & =10, \eta=100, \varepsilon_{i}=0.01,(1 \leq i \leq 13), \\
\alpha & =1, \beta=2, a=1, b=-1, \gamma=2,
\end{aligned}
$$

for example, with the aid of Mathematica, we easily verify that

$$
\begin{aligned}
\tilde{c_{1}}= & -0.580816-23(1-\ell), \\
\tilde{c_{2}}= & 13.4295+5 g(t)+0.611833 \ell \\
& -0.300916 \ell^{2}-\frac{(4.72653-2 h) E(0)^{2} C_{p}^{6}}{\ell^{2}}, \\
\tilde{c_{3}}= & -0.295408+0.530816 c^{2}+\frac{h}{8}+0.01 \ell, \\
\tilde{c_{4}}= & -\left(5100.03+50 c^{2}\right)(1-\ell)-5 \xi-100 \xi g(0) .
\end{aligned}
$$

Taking another example into consideration,

$$
\begin{gathered}
M=10, \eta=10, \varepsilon_{i}=\frac{i}{1000},(1 \leq i \leq 13), \\
\alpha=1, \beta=2, a=1, b=-1, \gamma=2,
\end{gathered}
$$

we find that$$
\tilde{c_{2}}=-1183.8+5 g(0)+2.464 \ell-1.2255 \ell^{2}
$$$$
-\frac{(38.8695-2 h) E(0)^{2} C_{p}^{6}}{\ell^{2}},
$$$$
\tilde{c_{3}}=-2.43435+4.81469 c^{2}+\frac{h}{8}+0.012 \ell,
$$$$
\tilde{c_{4}}=-\left(1321.45+83.3333\left(c^{2}+C_{p}\right)\right)(1-\ell)-5 \xi
$$$$
-25 \xi g(0)-73.2323 C_{p} g(0) \xi .
$$

Additionally, we may retrieve more effective data of $\tilde{c}_{i}$, and the data provide an intuitive understanding in the discussion of the auxiliary functionals.

Considering the equivalence between $L(t)$ and $\varepsilon(t)$, it is easy for us to see that

$$
M_{1} \varepsilon(t) \leq L(t) \leq M_{2} \varepsilon(t),
$$

where the coefficients $M_{1}$ and $M_{2}$ in (53) have many possibilities to be chosen.

The following expression can be presented through the combination of (48) and (53):

$$
L^{\prime}(t) \leq-\frac{h}{M_{1}} \varepsilon(t) .
$$

After a simple integral on the interval $\left(t_{0}, t\right),(54)$ leads to

$$
L(t) \leq e^{-\left(h\left(t-t_{0}\right) / M_{1}\right)} L\left(t_{0}\right) .
$$

As a consequence, taking $k=-\left(h / M_{1}\right)$, (55) leads to

$$
\varepsilon(t) \leq \frac{L\left(t_{0}\right)}{M_{1} e^{\left(h / M_{1}\right)\left(t-t_{0}\right)}} \leq \frac{K_{1} \varepsilon(0)}{e^{k t}},
$$

where $K_{1}$ is some appropriate positive constant. 
Case 2. $p>1$.

Using $H_{1}, H_{2}$, and (9), we easily verify that

$$
\int_{0}^{\infty} g^{1-\theta}(\zeta) \mathrm{d} \zeta \leq \int_{0}^{+\infty}\left(\frac{1}{d_{1} t+d_{2}}\right)^{(1-\theta / p-1)} \mathrm{d} \zeta<\infty, \quad 0 \leq \theta<2-p .
$$

Moreover, considering the inequality in [6], for some constant $C$,

$$
g \circ \nabla u \leq C\left(E(0) \int_{0}^{\infty} g^{1-\theta}(\zeta) \mathrm{d} \zeta\right)^{(p-1 /(p-1+\theta))}\left(g^{p} \circ \nabla u\right)^{(\theta / p-1+\theta)} .
$$

Taking $\theta=(1 / 2), \gamma=2 p-1$, we find $(\gamma \theta / p-1+\theta)=1$. Therefore, for some $m>1$, we get

$$
\varepsilon^{m}(t) \leq C\left(\int_{\Omega}|u|^{2} \mathrm{~d} x+\int_{\Omega}|\nabla u|^{2} \mathrm{~d} x+\int_{\Omega}\left|u_{t}\right|^{2} \mathrm{~d} x+g^{p} \circ \nabla u\right),
$$

that is,

$$
-\left(\int_{\Omega}|u|^{2} \mathrm{~d} x+\int_{\Omega}|\nabla u|^{2} \mathrm{~d} x+\int_{\Omega}\left|u_{t}\right|^{2} \mathrm{~d} x+g^{p} \circ \nabla u\right) \leq-\frac{1}{C} \varepsilon^{m}(t) .
$$

Taking (48) into account, we have

$$
-\varepsilon^{m}(t) \leq-\frac{1}{M_{2}^{m}} L^{m}(t)
$$

For each $t \geq t_{0}$, it is straight for us to get

$$
\begin{aligned}
L^{\prime}(t) & \leq \frac{h}{\gamma+2} \int_{\Omega}|u|^{\gamma+2} \mathrm{~d} x+L^{\prime}(t) \\
& \leq-\frac{h}{2}\left(\int_{\Omega}\left|u_{t}\right|^{2} \mathrm{~d} x+\ell \int_{\Omega}|\nabla u|^{2} \mathrm{~d} x+c^{2} \int_{\Omega}|u|^{2} \mathrm{~d} x+g \circ \nabla u\right) \\
& \leq-\frac{h}{C} \varepsilon^{m}(t) \\
& \leq-\frac{h}{C M_{2}^{m}} L^{m}(t) \\
& \leq-C L^{m}(t) .
\end{aligned}
$$

As we mentioned before, the symbol $C$ denotes different constants in different places. Executing a simple integral on the interval $\left(t_{0}, t\right),(62)$ yields

$$
L(t) \leq\left(C_{a} t+C_{b}\right)^{-(1 / m-1)}=\left(C_{a} t+C_{b}\right)^{-(1 / 2(p-1))} .
$$

Considering the equivalence between $L(t)$ and $\varepsilon(t)$, it is apparently for us to get

$$
\varepsilon(t) \leq \frac{K_{2}}{(t+1)^{(1 / 2(p-1))}} .
$$

This completes the proof.

\section{Conclusion}

Under the assumptions on the relaxation function and the interval of the fuzzy number $\eta$, applying the computational technique, a lot of auxiliary functionals can be constructed numerically. Two decay results, the exponential one and the polynomial one, are derived for the model (1) eventually. The result shows a new way for the decay rates, which is quite different from other literatures.

\section{Data Availability}

All datasets generated for this study are included in the manuscript.

\section{Conflicts of Interest}

The author declares that there are no conflicts of interest.

\section{Acknowledgments}

This work was supported by the Youth Foundation of Jining University (2019QNKJ03).

\section{References}

[1] S. Berrimi and S. A. Messaoudi, "Existence and decay of solutions of a viscoelastic equation with a nonlinear source," Nonlinear Analysis: Theory, Methods \& Applications, vol. 64, no. 10, pp. 2314-2331, 2006.

[2] S. Berrimi and S. A. Messaoudi, "Exponential decay of solutions to a viscoelastic equation with nonlinear localized damping," Electron Journal of Differential Equations, vol. 88, pp. 1-10, 2004.

[3] M. M. Cavalcanti and H. P. Oquendo, "Frictional versus viscoelastic damping in a semilinear wave equation," SIAM Journal on Control and Optimization, vol. 42, no. 4, pp. 1310-1324, 2003.

[4] S. A. Messaoudi and N.-E. Tatar, "Exponential and polynomial decay for a quasilinear viscoelastic equation," Nonlinear Analysis: Theory, Methods \& Applications, vol. 68, no. 4, pp. 785-793, 2008.

[5] F. Li and Y. Bai, "Uniform decay rates for nonlinear viscoelastic marguerre-von Kármán equations," Journal of Mathematical Analysis and Applications, vol. 351, no. 2, pp. 522-535, 2009.

[6] F. Li, "Limit behavior of the solution to nonlinear viscoelastic marguerre-von Kármán shallow shell system," Journal of Differential Equations, vol. 249, no. 6, pp. 1241-1257, 2010.

[7] F. Li, Z. Zhao, and Y. Chen, "Global existence uniqueness and decay estimates for nonlinear viscoelastic wave equation with boundary dissipation," Nonlinear Analysis: Real World Applications, vol. 12, no. 3, pp. 1759-1773, 2011.

[8] F. S. Li and W. M. Zhu, "Optimized uniform decay estimate of the solution to petrovsky equation with memory," Applied Mathematics and Optimization, vol. 20, 2020.

[9] M. L. Santos, M. M. Freitas, and A. J. A. Ramos, "Blow-up result and energy decay rates for binary mixtures of solids with nonlinear damping and source terms," Nonlinear Analysis, vol. 52, pp. 1468-1218, 2020.

[10] F. Y. Zhang, F. S. Li, and X. P. Chen, "Uniform decay rates for the viscoelastic Klein-Gordon equation," Applied Mathematics (Wuhan).vol. 32, no. 2, pp. 262-271, 2019. 
[11] H. Liu, Y. Pan, S. Li, and Y. Chen, "Adaptive fuzzy backstepping control of fractional-order nonlinear systems," IEEE Transactions on Systems, Man, and Cybernetics: Systems, vol. 47, no. 8, pp. 2209-2217, 2017.

[12] H. Liu, S. Li, H. Wang, and G. Li, "Adaptive fuzzy synchronization for a class of fractional-order neural networks," Chinese Physics B, vol. 26, no. 3, Article ID 030504, 2017.

[13] L. C. Evans, "Partial differential equations," in Graduate Student in Mathematics)ournal of the American Mathematical Society, Providence, RI, USA, 1998. 